\title{
Semiconductor Optical Amplifier-Fiber Laser and Its Improvement for Sensor Application
}

\author{
Yoshitaka TAKAHASHI, ${ }^{1}$ Ryo NIIDA, ${ }^{1}$ and Hiromu OTANI ${ }^{2}$ \\ ${ }^{1}$ Department of Electronic Engineering, Graduate School of Engineering, Gunma University, \\ 1-5-1 Tenjin-cho, Kiryu, Gunma 376-8515 \\ ${ }^{2}$ Mitsuba Corporation, 1-2681 Hirosawa-Cho, Kiryu, Gunma 376-8555
}

(Received January 31, 2008)

\begin{abstract}
We have developed a novel optical fiber ring laser using a semiconductor optical amplifier (SOA) as the gain medium, and taking advantage of polarization anisotropy of its gain. Since the frequency difference of the bi-directional laser is proportional to the introduced birefringence, the laser in the present study is applicable to a frequency-domain fiber sensor with the birefringent material. For the application the accuracy and stability of the beat signal frequency is important, but it was not sufficient because the polarization of the propagating light was not constant in the single-mode fiber (SMF) which was incorporated into the ring laser cavity. In order to reduce this ambiguity, we have improved the fiber ring laser by using polarization-maintaining fiber (PMF) and a PMF-coupler instead of SMF and a SMF-coupler. The linewidth of the beat signal has been narrowed and the accuracy of the beat signal frequency has been enhanced.
\end{abstract}

Key Words: Fiber laser, Fiber sensor, Ring laser, Semiconductor optical amplifier, Optical sensor

\section{Introduction}

Optical fiber sensors are widely used in various uses since it has many advantages. Most of them detect an optical intensity change and if there is fluctuation of the source intensity and/or a propagation loss in the sensing system it will cause measurement errors. Sensors which are not influenced by the fluctuation have been required and studied, e.g. optical heterodyne interferometry, a frequency-domain sensor, and so on. We have developed and studied a novel optical fiber ring laser $^{1-3)}$ using a semiconductor optical amplifier (SOA) as the gain medium, which has polarization anisotropy as well as many advantages. In general cases birefringence applied to a ring laser is reciprocal effect and no phase difference generates between two counterpropagating lights. Fiber ring lasers for frequency-domain sensors were proposed, e.g., an optical fiber gyro $^{4,5)}$ using Sagnac effect and an optical current sensor ${ }^{6,7)}$ using Faraday effect. These effects are non-reciprocal effect. In the present study taking advantage of the polarization anisotropy of SOA, we control the phase difference, and then the oscillation frequency difference between two counter-propagating lights by incorporating a birefringent medium into the ring cavity. Since the frequency difference is proportional to the birefringence, the laser in this study is applicable to a novel fiber sensor of which the sensing signal is obtained in a frequency domain with the material which causes the retardation change by a physical phenomenon to be measured. There are many phenomena such as electromagnetic field, pressure, and temperature, which cause birefringence, sensors utilizing the present fiber ring lasers are expecting to detect such physical quantity in a frequency domain. In investigating the sensor application, it was found that the accuracy and stability of the beat signal resulting from the frequency difference, which was important for the application, was not sufficient because the polarization of the propagating light was not constant in the single-mode fiber (SMF) which was incorporated into the ring laser cavity. In order to reduce this ambiguity, the authors have improved the fiber ring laser by using polarization-maintaining fiber (PMF) and a PMF-coupler instead of SMF and a SMF-coupler.

\section{Principle of Operation}

The operating principle of the SOA ring laser ${ }^{1)}$ is shown in Fig. 1. SOA is the gain medium of the laser. It has polarization anisotropy of its gain and is regarded as an amplifier only for TE-polarization, which is assumed to be in accordance with

the horizontal plane. The ring cavity contains Faraday rotators $R_{1}$ and $R_{2}$ which generate $+45^{\circ}$ and $-45^{\circ}$-rotation of the state of polarization (SOP) of light propagating in the

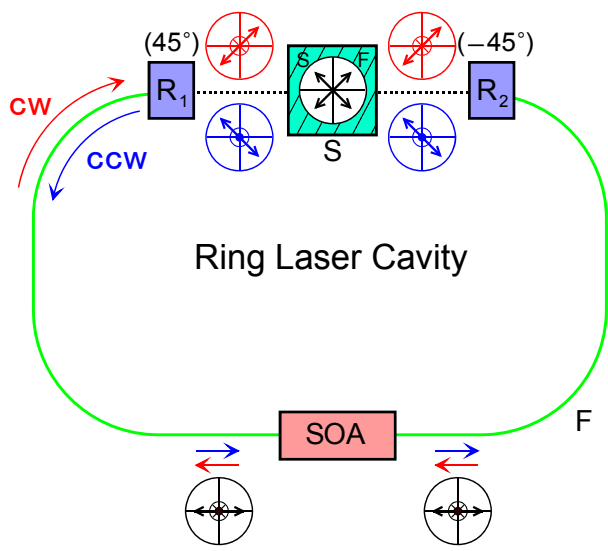

Fig. 1 Schematic diagram of SOA-fiber ring laser. The red and blue arrows in circles represent the state of polarization (SOP) of the cw and ccw lights, respectively. 
clockwise (cw) direction. The cavity also contains a medium S which has birefringence (retardation $R$ ) and works as a sensing element when the laser is applied to a fiber sensor.

First, consider light which propagates in the cw direction in the ring cavity. Horizontally polarized light emitted from SOA is coupled into the cavity and passes through $\mathrm{R}_{1}$, and, as a result, SOP of the cw light rotates $45^{\circ}$ (red arrow in a circle shown in Fig. 1). The dielectric axes (F-axis and S-axis) of the birefringent medium $\mathrm{S}$ are at an angle of $45^{\circ}$ to the horizontal plane. The $\mathrm{cw}$ light, therefore, passes through $\mathrm{S}$ with the polarization plane in accordance with the F-axis. The cw light turns horizontally polarized after passing through another Faraday rotator $\mathrm{R}_{2}$, and returns to SOA with the polarization corresponding to its TE-polarization.

On the other hand, the counterclockwise (ccw) light emitted from SOA passes through $S$ with the polarization plane in accordance with the S-axis because of the nonreciprocity of Faraday effect, and returns to SOA with the polarization corresponding to its TE-polarization in a similar way. That is, the $\mathrm{cw}$ and $\mathrm{ccw}$ lights have orthogonal polarization to each other in passing through $\mathrm{S}$, while they have the same polarization in other regions and are both amplified by SOA. Thus phase difference is brought about between the $\mathrm{cw}$ and ccw lights by retardation in S, and causes them to oscillate in different frequencies. The frequency difference $f_{B}$ is proportional to the retardation $R[\mathrm{~nm}]$ and given by ${ }^{8)}$

$$
\begin{aligned}
f_{B} & =\frac{c}{\lambda L} R \\
& =\frac{R}{\lambda} f_{F S R}
\end{aligned}
$$

where $\lambda[\mathrm{nm}]$ is the lasing wavelength, $c$ is the velocity of light, $L$ and $f_{F S R}=c / L$ is the optical path length and the free spectral range of the ring cavity, respectively. If $S$ is the medium which causes the retardation change by the physical phenomenon to be measured, the change can be detected by measuring $f_{B}$ and the laser in this study can be applied to a sensor which detects the signal in a frequency domain.

\section{Configuration}

\subsection{SOA-Fiber Ring Laser with SMF (FRL-SMF)}

Figure 2 shows the configuration of the fiber ring laser in the early stage without PMF. The emitted lights at $1.55 \mu \mathrm{m}$ from both sides of SOA (Anritsu Corp.) were coupled into singlemode fibers (SMF) with singlemode ball-lens fibers (BLF), collimated with fiber-collimators $\left(\mathrm{C}_{1}, \mathrm{C}_{2}\right)$ and then passed through a sensing element (S) in the space-propagating region. At the ends of the two fiber-collimators Faraday rotators $\left(\mathrm{R}_{1}, \mathrm{R}_{2}\right) 0.7 \mathrm{~mm}$ thick were attached. They were magnetized and generated $45^{\circ}$-rotation of the polarization angle of the $1.55 \mu \mathrm{m}$ light without an external magnet. Both the $\mathrm{cw}$ and $\mathrm{ccw}$ lights of the ring laser were output via a 10 $\mathrm{dB}$-singlemode fiber directional coupler $\left(\mathrm{FC}_{1}\right)$, and in order to examine the frequency difference they were combined with each other via a $3 \mathrm{~dB}$-singlemode fiber directional coupler $\left(\mathrm{FC}_{2}\right)$ and detected by an avalanche photodiode (APD). Using two fiber polarization controllers (FPC) the SOP of both $\mathrm{cw}$ and ccw lights were adjusted in accordance with the TE-polarization of SOA, while they were linearly polarized in the space-propagating region. Two Faraday rotators $\left(R_{1}, R_{2}\right)$

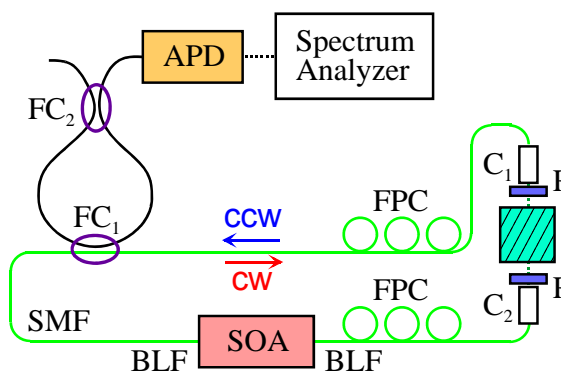

Fig. 2 SOA-fiber ring laser with SMF.

were placed complementarily as described before and thus the $\mathrm{cw}$ and $\mathrm{ccw}$ lights propagated in the orthogonal polarization to each other in passing through $\mathrm{S}$.

\subsection{SOA-Fiber Ring Laser with PMF (FRL-PMF)}

Figure 3 shows the configuration of the improved fiber ring laser with PMF. A ring cavity of the laser is comprised of SOA, BLF, SMF, PMF, $\mathrm{R}_{\mathrm{i}}$ 's (Faraday rotators), Sensing Element, and $\mathrm{C}_{i}$ 's(collimators). The dimension of fibers in Fig. 3 is not proportional to the actual length, and the ratio of the length of PMF to the total cavity length is $71.5 \%(10.8 \mathrm{~m} / 15.1 \mathrm{~m})$. The bidirectional laser output is coupled out by a PMF-coupler and combined by a SMF-coupler. Most optical elements are the same as in the previous laser except the ones related to PMF.

\section{Experiment}

The frequency difference $f_{B}$ is generated by a sensing element $S$ and observed as a beat in the power spectrum of APD signal. For sensor application, a frequency shift of the beat is measured to calculate the birefringence of S. Figure 4 shows the dependence of the frequency shift on applied retardation observed in the previous study ${ }^{1)}$ with FRL-SMF. The experimental data are plotted as circles, and though linear dependency is observed there is some erratic variation. In order to investigate its origin, the effect has been estimated numerically that results from some parameters.

4.1 Wavelength dispersion

Range of the oscillating wavelength was around 1542 to $1548 \mathrm{~nm}$. Faraday rotation and birefringence of waveplates are for $1550 \mathrm{~nm}$ and the maximum deviation is about $8 \mathrm{~nm}$. The resultant wavelength dispersion was calculated as:

Birefringence : $8 \mathrm{~nm} / 1550 \mathrm{~nm} \sim 0.5 \%$

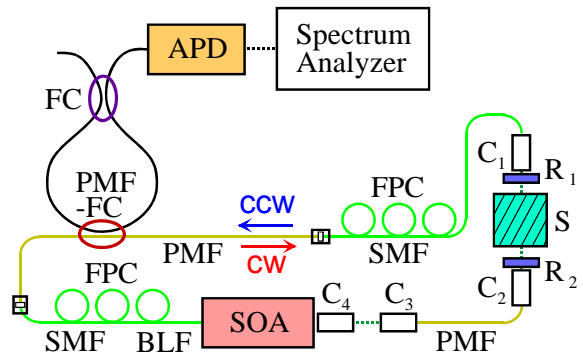

Fig. 3 SOA-fiber ring laser with PMF. 


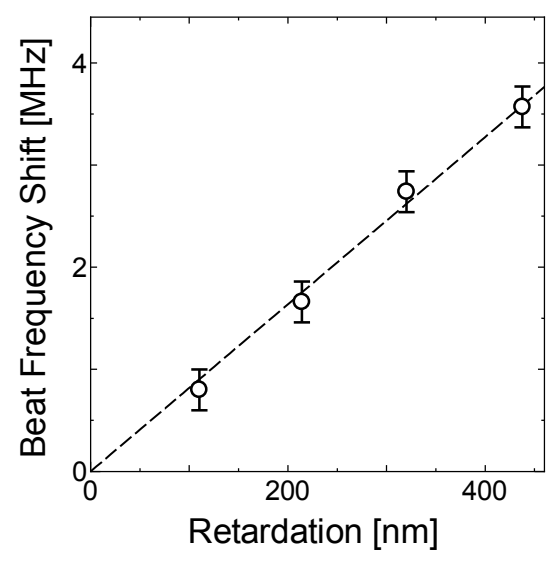

Fig. 4 Beat frequency shift with increasing retardation1).

Faraday rotation : $0.063^{\circ} / \mathrm{nm}(\mathrm{spec}) \times 8 \mathrm{~nm} / 45^{\circ} \sim 1.1 \%$

\subsection{Angle deviation}

Misalignment definitely causes errors and temperature dependence of optical elements would not be negligible. Temperature dependence of Faraday rotator is $0.09^{\circ} / \mathrm{K}$ (spec) and assuming $\pm 10 \mathrm{~K}$ change overall errors of Faraday rotation angle with the effect of wavelength dispersion is estimated as:

Faraday rotation angle : $\pm 1.5^{\circ}$.

This causes angle deviation of the waveplates $\eta$ as well as their misalignment. With the angle deviation $\eta$ the effective birefringence $\phi$ would change as

$$
\phi \rightarrow 2 \tan ^{-1}\left(\cos 2 \eta \tan \frac{\phi}{2}\right) .
$$

The calculated result was shown in Fig. 5 and errors estimated at $\phi=45^{\circ}$ was within a few percents.

By numerical analysis so far the calculated effects are not serious, which indicates that other parameters should be examined.

\subsection{Cavity instability}

The ring cavity includes some parts which are sensitive to misalignment, e.g. BLF coupled with SOA and the space-

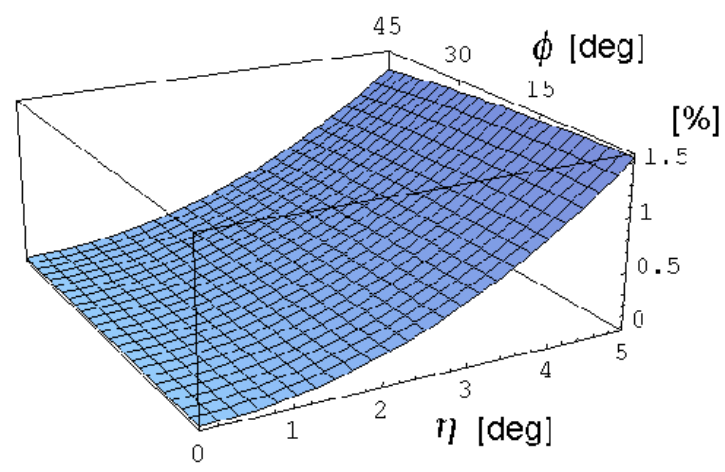

Fig. 5 Calculated errors originated from angle deviation $\eta$ of birefringence. (a)

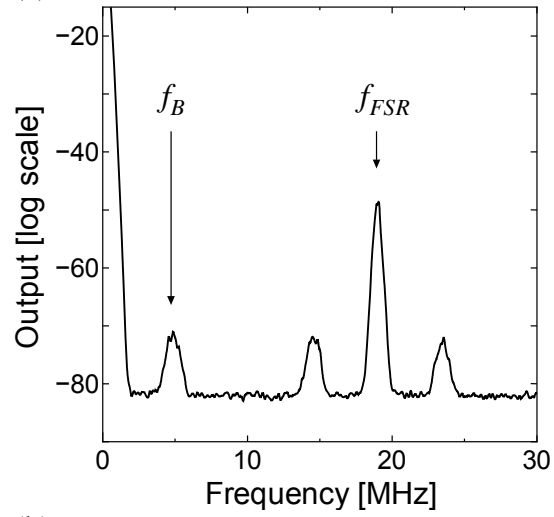

(b)

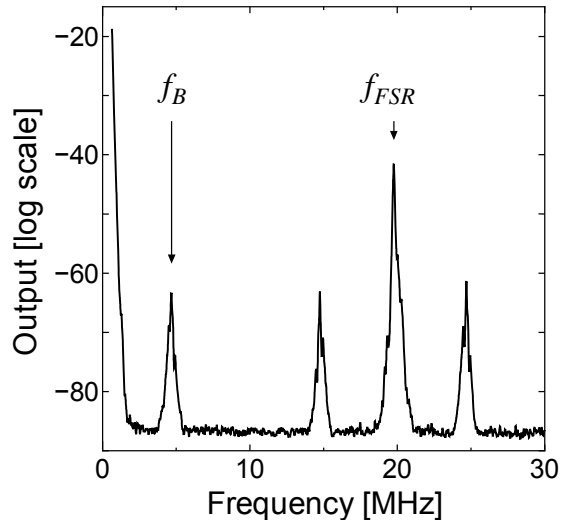

Fig. 6 Power spectrum: (a) FRL-SMF and (b) FRL-PMF.

propagating region. Since the laser has made use of the polarization anisotropy of SOA, polarization instability of the propagating light would also affect errors significantly though SOA would work as a polarizer. Numerical estimation of these effects is rather difficult. Consequently introduction of PMF in the cavity was decided as shown in Fig. 3 in addition to deliberate alignment. The spectra are shown in Fig. 6(a) FRL-SMF and Fig. 6(b) FRL-PMF in the case that a $\lambda / 4$ plate for $1550 \mathrm{~nm}$ was inserted as $\mathrm{S}$. Calculated with the corresponding cavity length, $f_{F S R}$ is (a) $18.6 \mathrm{MHz}$ and (b) 19.8 $\mathrm{MHz}$, respectively, and the retardation of $\mathrm{S}$ is $\lambda / 4$ and $f_{B}$ is, therefore, calculated into $1 / 4 f_{F S R}$ from (1). In Fig. 6 in addition to $f_{B}$ signal, $f_{F S R}$ signal with subsidiary signals that correspond to the beats between the counter-propagating oscillations with different mode numbers, which shows both lasers are in multimode operation. The linewidth of both $f_{B}$ and $f_{F S R}$ signals became significantly narrower because of the improvement of the polarization stability. The linewidth (FWHM) of $f_{B}$ varied from $0.89 \mathrm{MHz}$ to $0.07 \mathrm{MHz}$.

\section{Conclusion}

For sensor application the SOA-fiber ring laser has been developed. After some experiments and numerical analysis, polarization of the propagating light in the cavity should be stabilized though SOA would act as a polarizer. The laser was improved by introducing PMF instead of SMF. As shown in Fig. 6, since the polarization of propagating light becomes 
stable, the linewidth of the beat signal has been narrowed and the accuracy of the beat signal frequency has been enhanced. Judging from enhancement factor, the accuracy would improve more than one order. In the cavity of the improved fiber ring laser SMF is still incorporated and its replacement with PMF as shown in Fig. 7 will make the accuracy enhanced more.

\section{References}

1) Y. Takahashi, S. Sekiya, and N. Iwai: Opt. Rev. 10 (2003) 315.
2) Y. Takahashi, H. Kuroda, and T. Suemune: The Review of Laser Engineering 33 (2005) 329 (in Japanese).

3) T. Suemune and Y. Takahashi: Opt. Laser Eng. 45 (2007) 789.

4) R. K. Kadiwar and I. P. Giles: Electron. Lett. 25 (1989) 1729.

5) S. K. Kim, H. K. Kim, and B. Y. Kim: Opt. Lett. 19 (1994) 1810.

6) A. Kung, P. -A. Nicati, and P. A. Robert: IEEE Photon. Technol. Lett. 8 (1996) 1680.

7) Y. Takahashi and T. Yoshino: J. Lightwave Technol. 17 (1999) 591.

8) Y. Takahashi and T. Yoshino: Appl. Opt. 36 (1997) 6770. 